\title{
Archéopages
}

Archéopages

Archéologie et société

34 | 07/2012

Campagnes

\section{Les habitats ruraux du haut Moyen Âge en Seine- Saint-Denis. État des lieux}

Cristina Gonçalves-Buissart, Ivan Lafarge et Cyrille Le Forestier

\section{(2) OpenEdition}

1 Journals

Édition électronique

URL : https://journals.openedition.org/archeopages/394

DOI : 10.4000/archeopages.394

ISSN : 2269-9872

Éditeur

INRAP - Institut national de recherches archéologiques préventives

Édition imprimée

Date de publication : 1 février 2012

Pagination : 48-57

ISSN : 1622-8545

\section{Référence électronique}

Cristina Gonçalves-Buissart, Ivan Lafarge et Cyrille Le Forestier, "Les habitats ruraux du haut Moyen

Âge en Seine-Saint-Denis. État des lieux », Archéopages [En ligne], 34 | 07/2012, mis en ligne le 01

juillet 2012, consulté le 21 janvier 2022. URL : http://journals.openedition.org/archeopages/394 ; DOI : https://doi.org/10.4000/archeopages.394 


\section{Les habitats ruraux du haut}

Moyen Âge en Seine-Saint-Denis État des lieux

Cristina Gonçalves-Buissart Bureau du patrimoine archeologique dela Seine-Saint-Denis

Ivan Lafarge Burreau du patrimoine archeologique de la Seine-Saint-Denis, CH2ST-EA 227 «Centre dh histoire des techniques,

Cyrille Le Forestier Inrap

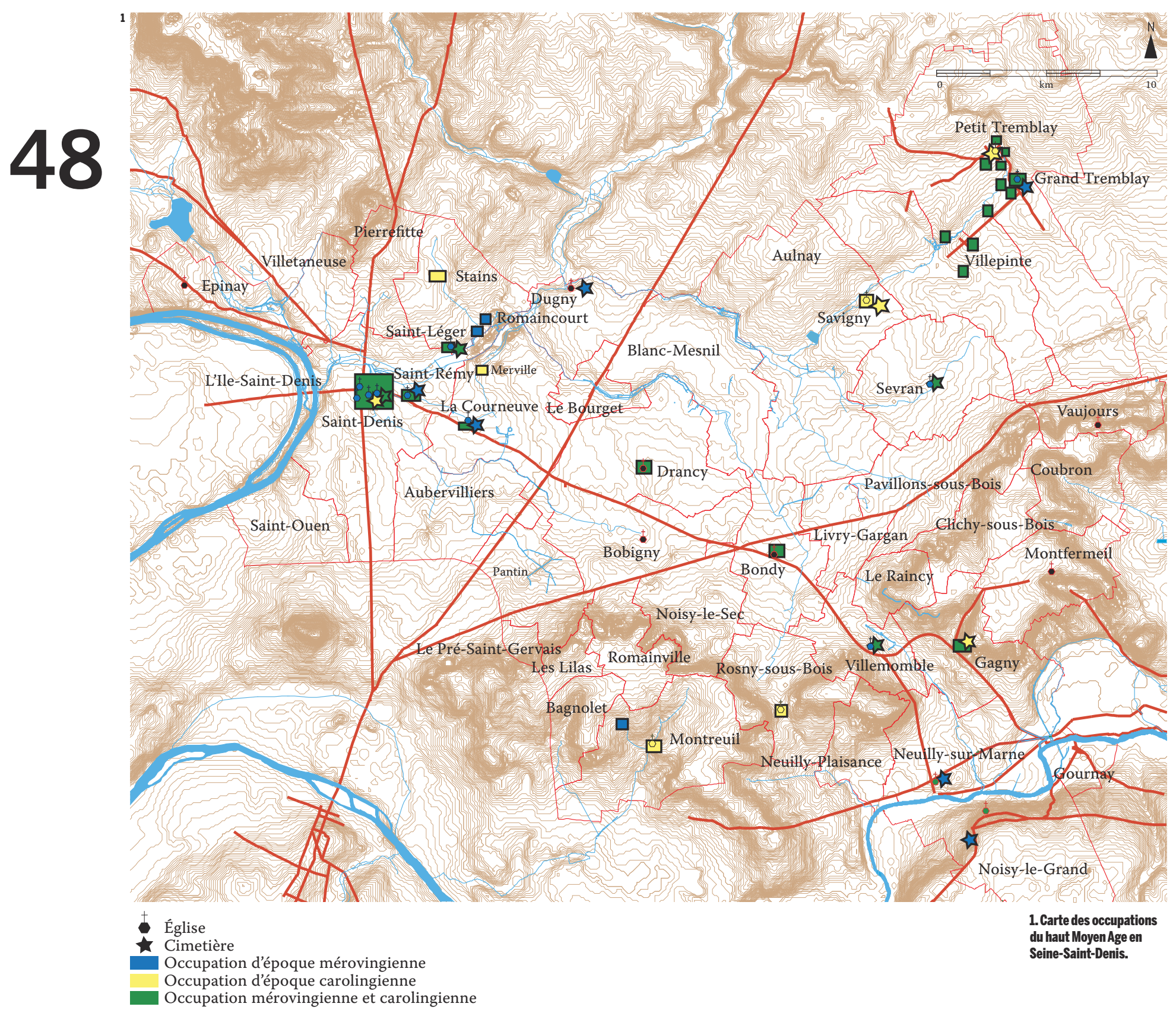


La Seine-Saint-Denis, parmi les départements de la petite couronne, de faible superficie et très urbanisés, est aujourd'hui celui où le haut Moyen Âge est le mieux représenté1. Il convient cependant de noter que la majorité des occupations mises au jour concernent des habitats ruraux et quelques nécropoles. Au travers des sites fouillés, on tente aujourd'hui d'appréhender les modes de fixation des habitats médiévaux et leur évolution à l'échelle départementale.

Le problème constamment rencontré sur le département est la taille des fenêtres d'observation: la plupart des opérations concernent des emprises inférieures à $10000 \mathrm{~m}^{2}$. La forte implication de l'équipe du Bureau de l'archéologie sur certaines communes, aux centres anciens marqués, notamment Tremblay-en-France, Drancy, Gagny permet de palier l'absence d'interventions sur de vastes terroirs et de tenter d'appréhender l'origine des villages. Force est de constater que mises bout à bout, les petites fenêtres d'observation autorisent l'esquisse d'une synthèse sur l'évolution des villages, le cas du Vieux Pays de Tremblay-en-France étant le plus abouti. Cet investissement est un choix de la collectivité qui a pris le parti de suivre tous les travaux d'aménagement dans ce village. Le but est la collecte d'un maximum d'informations à travers un panel varié d'interventions. La nature même des opérations et la taille réduite des emprises de décapage impliquent donc que l'image de ces sites reste incomplète tant au niveau spatial que chronologique. Les limites d'occupation ne sont ainsi que rarement connues. La faiblesse, voire l'absence, de relations stratigraphiques entre les structures ne permet pas non plus d'affiner les datations, d'autant que le mobilier se rattache presque toujours à la phase d'abandon des structures et non à celle de leur utilisation. Les archéologues du département participent au PCR sur l'habitat rural du haut Moyen Âge en Ile-de-France depuis ses débuts (Lefèvre et al., 2003 et 2009); les travaux menés à l'échelle du département depuis une vingtaine d'années ont une résonance notable avec ceux menés par d'autres membres du PCR tant en Val d'Oise qu'en Seine-et-Marne.

1. L’archéologie du haut Moyen Âge s'est développée en SeineSaint-Denis à la faveur de plusieurs facteurs. Tout d'abord, de nombreuses fouilles effectuées depuis les années 1970 et pendant les années 1980 par diverses associations concernent le haut Moyen Âge. Le sauvetage du centre urbain de Saint-Denis a engendré la création de l'Unité d'Archéologie de la ville de Saint-Denis en 1982. Depuis la mise en place du service départemental en 1991 le nombre des opérations et les données concernant le haut Moyen Âge en Seine-Saint-Denis n'a cessé de s'accroître.

\section{diversifié} (de 20 à $50 \mathrm{~m}$ ), structuré par les larges vallées de la Seine et de la Marne et surmonté par un relief de buttes-témoins : le plateau de l'Aulnoye (130 m), le plateau de Romainville-Montreuil (128 m) et le plateau d'Avron $(115 \mathrm{~m})$. Ces buttes se rattachent morphologiquement à l'extrémité occidentale du plateau de la Brie. Elles sont marquées par les dépressions de Gagny et de Rosny-sous-Bois, vestiges de la pré-Seine. Une autre limite paléogéographique dans l'axe de la vallée de la Marne marque le paysage départemental : la limite entre les formations bartonniennes du gypse vers le nord ouest et les calcaires lacustres de Brie vers le sud-est. Au nord-ouest du département se trouve la Butte-Pinson, qui annonce les premiers contreforts du plateau de Montmorency.

Parmi les ressources naturelles de ce territoire, le gypse sert à la fabrication du plâtre et le limon a largement été exploité pour la terre cuite architecturale. Le calcaire est disponible au sud, à Paris, dans la vallée de la Seine en général et au nord sur le plateau du Parisis, jusquà la vallée de l'Oise. Ce territoire marqué par des terrains calcaires affleurants (marnes et Saint-Ouen) ou des formations superficielles de plateaux (loess) est propice à l'agriculture céréalière dans les zones de plateau, maraîchère et horticole sur les buttes et dans les zones basses, ainsi qu'à l'élevage.

\section{Réseaux hydrographique et viaire fixateurs des habitats}

Le réseau hydrographique de la Seine-SaintDenis est marqué par la présence d'un méandre de la Seine à l'ouest et de la Marne au sud-est. Le territoire est également sillonné par de nombreux ruisseaux, qui correspondent pour la plupart à la moitié sud du bassin versant du Croult qui débouche dans la Seine à Saint-Denis. Ce réseau secondaire est riche en ressources complémentaires, et joue un rôle fixateur des habitats dès la Préhistoire. Létude des cartes et plans anciens révèle un territoire sillonné de nombreux itinéraires dont l'ancienneté est confirmée pour bon nombre de tracés. Les voies dessinent un réseau en étoile autour de Paris vers Rouen (chaussée Jules César), Amiens, Senlis, Meaux... mais aussi un itinéraire de grand parcours reliant les vallées de la Seine et de la Marne sans passer par Paris, fossilisé par la RN186 et une portion de l'autoroute A86. La majorité des occupations alto-médiévales attestées se trouvent en bordure d'axes importants de circulation [ill. 1].

\section{Structures de l'habitat rural}

Les structures de combustion sont des structures d'activités sur lesquelles la nature même de l'occupation a une influence : sur le statut (familial ou collectif) et sur leur organisation. Il est donc important de prendre en compte toutes les structures de cuisson (Lefèvre et al., 2003 et Lefèvre et al., 2009). Une attention particulière est portée aux petits foyers isolés, plutôt mal conservés, mais présentant des soles très épaisses, attestés sur quelques sites séquano-dyonisiens (notamment Drancy - rue LouisDelplacé ou Tremblay-en-France - rue Jules-Guesde) et fréquents au début de la période mérovingienne. Les sites les plus riches en fours domestiques (ceux-ci étant en excellent état de conservation) sont situés à Tremblay-en-France avec notamment ceux de la RD4o (24 fours) et la rue Jules-Guesde (44 fours). Le premier a livré un ensemble de trois fours qui s'organisent autour d'une même fosse. Ces fours ont conservé leur voûte en place avec tuyère, les traces des outils utilisés pour leur creusement en sape, des soles épaisses avec 


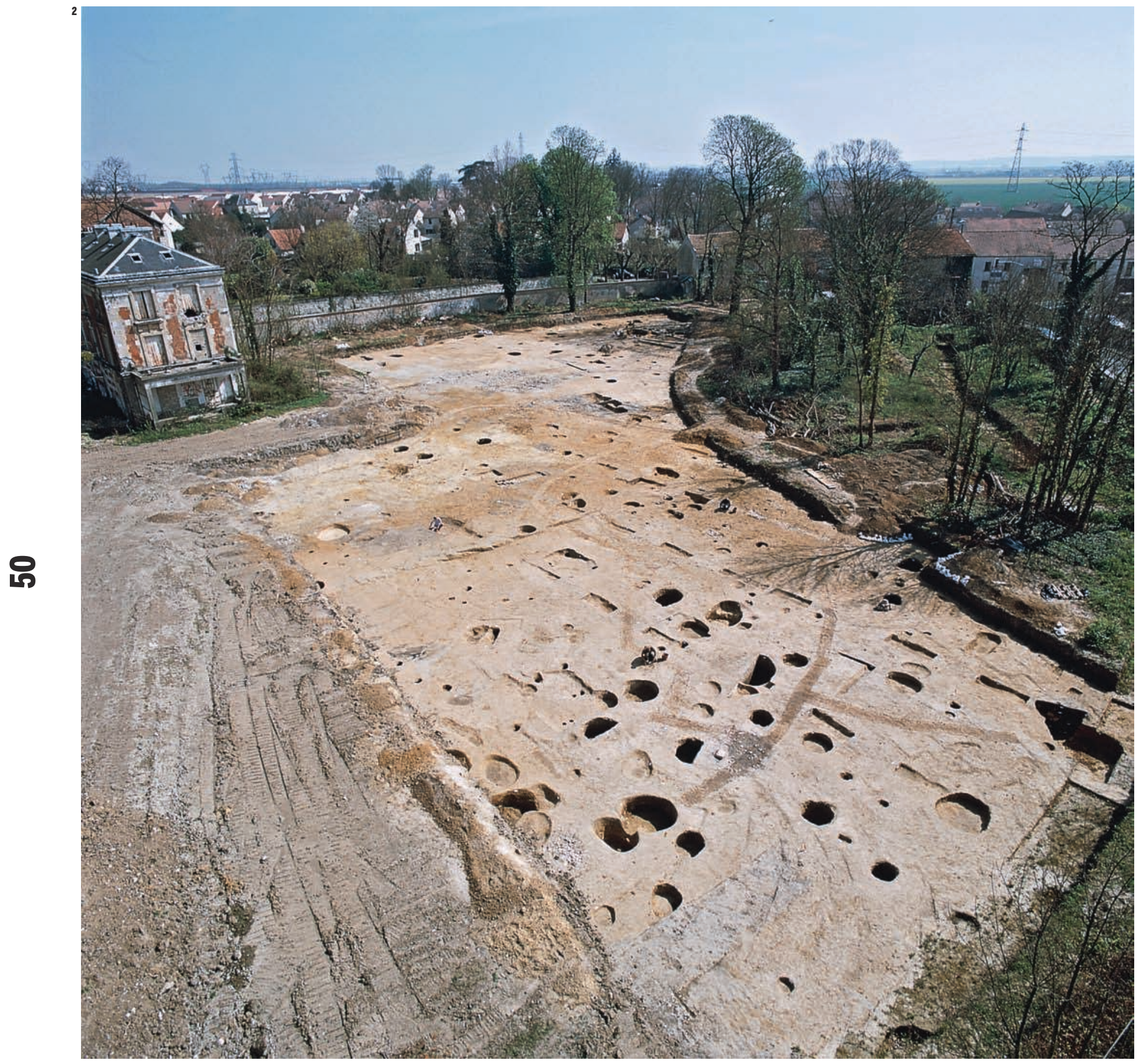

2.Vue générale de

la fouille de la rue des

Tilleuls à Tremblay-en-

France (2007). Cette

fouille montre des

vestiges caractéristiques

des sites d'habitat

du haut Moyen Âge en

Ile-de-France,

essentiellement fosses

et fonds de cabanes avec

une prédominance des

structures de stockage

l'occupation est continue

depuis le ve siècle avec

une forte densité de

structure des XI-XII siècles. 
un radier préalable en pierres et tuiles pour l'un d'entre eux et des niveaux de rejets de charbons. Le site de la rue Jules-Guesde est intéressant quant à lui, par la présence de nombreux ensembles de fours mérovingiens, mais également par la mise au jour de soles épaisses isolées, attribuées par archéomagnétisme, à la transition Bas Empire période mérovingienne. Tous les sites de même attribution chronologique, à Tremblay-en-France, ont livré des structures de combustion en nombre, fonctionnant seules ou en batterie. Pour d'autres sites, le nombre de structures de combustion est faible. Nous avons à faire plutôt à des foyers, souvent associés à des fonds de cabane.

Les fonds de cabane sont le plus souvent sur le mode à deux poteaux axiaux. L'autre type très représenté sur l'ensemble des sites de la Seine-SaintDenis mais également du nord de la France est celui à quatre poteaux corniers. Le site le plus représentatif et dont le corpus est le plus important (26) est celui de «l'Imprimerie du Figaro » à Tremblay-en-France. On en relève treize rue Jules-Guesde et douze sur le site de la RD40. À noter que pour l'ensemble des sites de Tremblay-en-France, les fonds de cabane, quel que soit le type, sont relativement arasés et de dimensions légèrement inférieures à celles observées sur d'autres sites d'Ile-de-France [ill. 2] (Serris, l'Arpent Ferret en Seine-et-Marne). Les aménagement internes sont peu nombreux et concernent essentiellement des fosses d'accès ou des fosses d'ancrage de métier à tisser. Les objets pouvant témoigner de leur activité sont ténus mais néanmoins existants (poinçons en os, fusaïole...).

Sur les quarante communes de Seine-Saint-Denis, dix-sept ont livré des sépultures mérovingiennes et carolingiennes. Si la plupart des nécropoles perdurent durant tout le haut Moyen Âge, seules quelques-unes succèdent sur le même lieu à une nécropole gallo-romaine (Saint-Denis, Bondy). Il est rare de constater un lien direct entre nécropole et habitat. Les quelques 2 ooo sépultures altomédiévales recensées en Seine-Saint-Denis se concentrent principalement dans des nécropoles mais il existe de rares sépultures dispersées (moins de 100 individus) présentes à Tremblay-en-France, Saint-Ouen, Dugny. Les individus sont parfois regroupés (3 à 5) comme sur les sites «l'Imprimerie du Figaro » ou celui de la RD4o à Tremblay-enFrance. Les pratiques funéraires sont similaires à celles des sujets des nécropoles. Les raisons de l'isolement de ces inhumations et le rapport qu'elles entretiennent avec les cimetières et les sites d'habitat restent toutefois difficiles à comprendre. Si le Concile de Tibur met en place dès 895 l'obligation de se faire enterrer dans le cimetière paroissial autour d'un lieu saint, il semble qu'une certaine liberté dans le choix du lieu d'inhumation subsiste après cette date. Ce choix peut être motivé par des raisons d'ordre financier (prix de la concession), d'ordre religieux (individus juifs ou non baptisés), ou par des motifs liées à l'âge ou au sexe des individus concernés (même si aucun recrutement spécifique n'a été réellement mis en avant dans les cas mentionnés), ou encore relever d'un choix personnel. Nous ne connaissons que deux cas avérés de sépultures de relégation : à Tremblayen-France, une femme repose dans un four, les mains dans le dos et la face contre terre ; à Bondy, deux jeunes de 8 à 12 ans ont été jetés dans un silo à demi rempli de graines.

\section{Architecture et matériaux de construction}

Létude architecturale et celle des matériaux de construction, ainsi que le développement d'analyses de plus en plus performantes et variées sur un panel de plus en plus large de vestiges, permettent de mieux cerner la diversité et la complexité du monde rural du premier Moyen Âge. Peu d'éléments éclairent efficacement la question de la construction pour le haut Moyen Âge sur notre territoire. On ne dispose que de rares bâtiments sur poteaux concordants avec ce qui ressort des exemples de comparaison régionales.

Plusieurs édifices de culte sont attestés entre le très haut Moyen Âge et l'époque carolingienne, soit par les sources, leur titulature ou les éléments morphotopographiques. La plupart de ces édifices en eux-mêmes n'est pas connu, quelquefois tout simplement parce que l'édifice actuel surmonte les vestiges des états antérieurs. On peut croire qu'une bonne partie d'entre eux a longtemps été des édifices en bois. C'est significatif de la quasi disparition du paysage monumental antique en pierre dès le début du haut Moyen Âge.

Les édifices de culte reconnus en Seine-SaintDenis rattachables à une période antérieure à lépoque carolingienne sont extrêmement rares : la basilique de Saint-Denis et les églises Saint-Barthélémy à Saint-Denis, Saint-Genès à Villemomble et SaintLucien à La Courneuve. Aucun bâtiment sur poteau ne peut pour le moment être assimilé à une église. Trois édifices sont clairement maçonnés à l'époque mérovingienne : l'abbatiale de Saint-Denis, l'église Saint-Barthélémy à Saint-Denis et l'église SaintGenès de Villemomble. Les maçonneries sont en moellons disposés en épis ou arrête de poisson opus spicatum et liés à la terre, au plâtre ou à la chaux. À La Courneuve, où les éléments observés permettent de conclure à l'existence d'un édifice, on n'est pas en mesure de trancher sur le mode constructif de l'église.

L'usage de la pierre durant le haut Moyen Âge est peu rationalisé et demeure rare dans la construction. L'aqueduc mérovingien de Saint-Denis, construit en dalles de calcaire grossier jointoyées en mortier de tuileau friable reste exceptionnel et est en lien avec l'ensemble abbatial. Une grande partie de la pierre de construction mérovingienne est issue de remplois, comme à la basilique de Saint-Denis, mais on constate pour les massifs de fondation et le moellonnage, la prédominance des pierres siliceuses, chailles, meulières, encroûtements, caillasses et calcaires de Saint-Ouen et des marnes du gypse, issues de ramassages de surface ou de creusements 


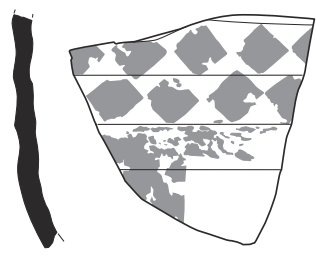

fine sombre polie

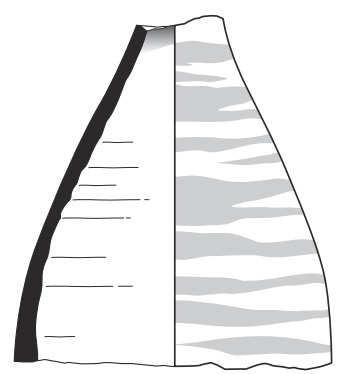

กิ

3. Planche de tessons de céramiques du haut Moyen Âge provenant des sites de Tremblay-enFrance, allée des Tilleuls

(Fouille en 2007) et

"l'Imprimerie du Figaro » (Fouille en 2008).
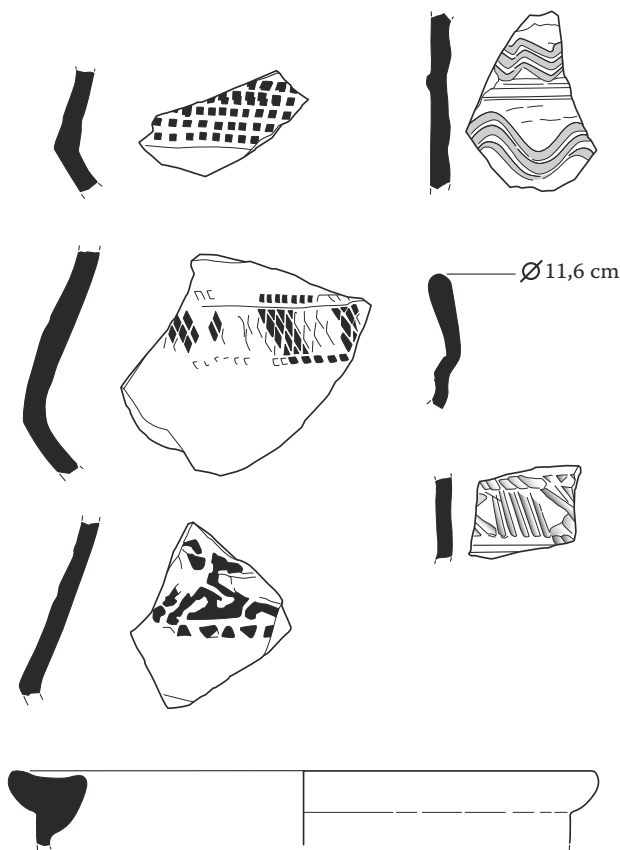

semi-fine peinte et polie

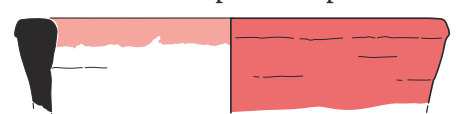

granuleuse à inclusions fines

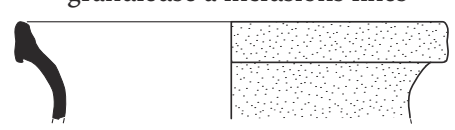

)
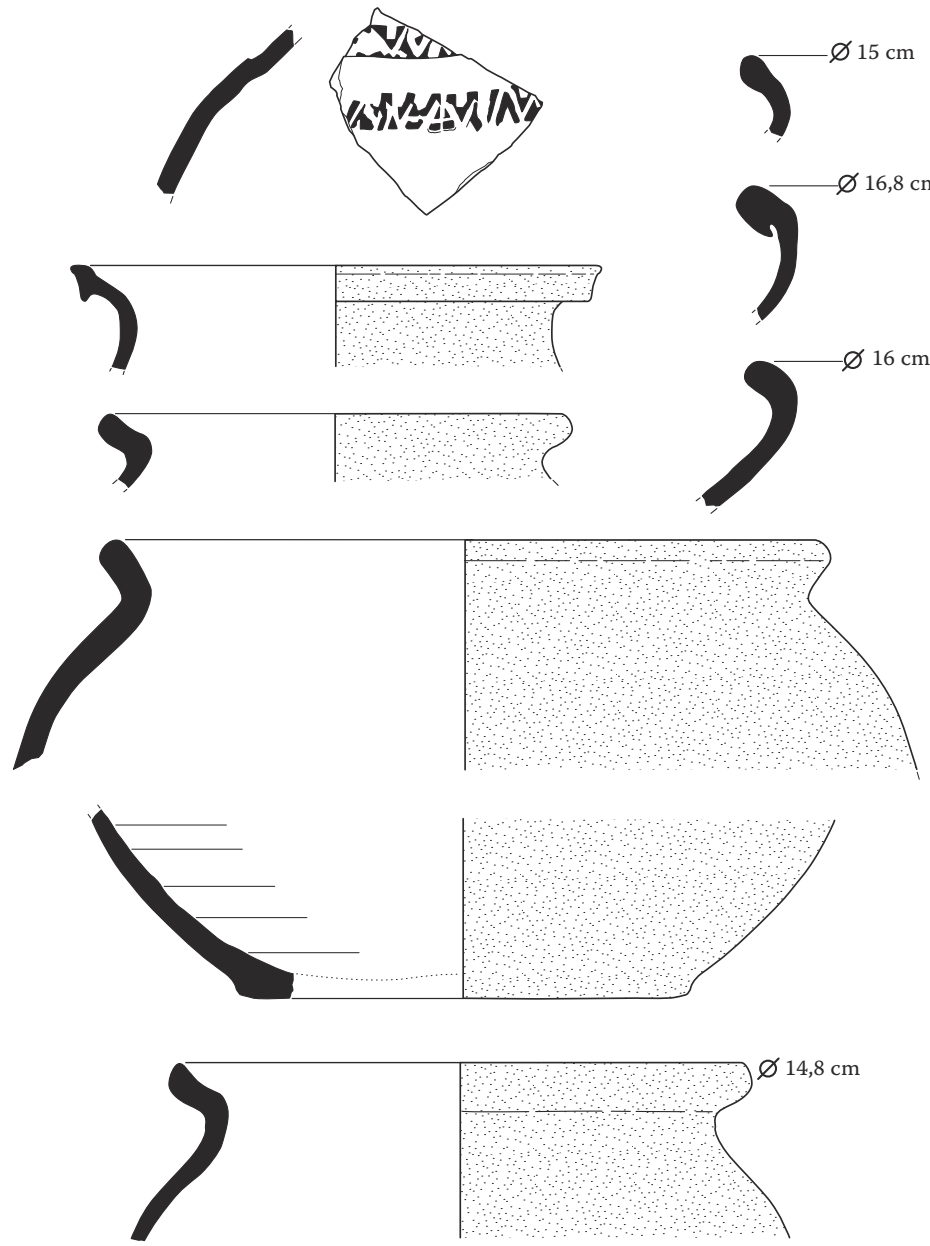

granuleuse à inclusions moyennes
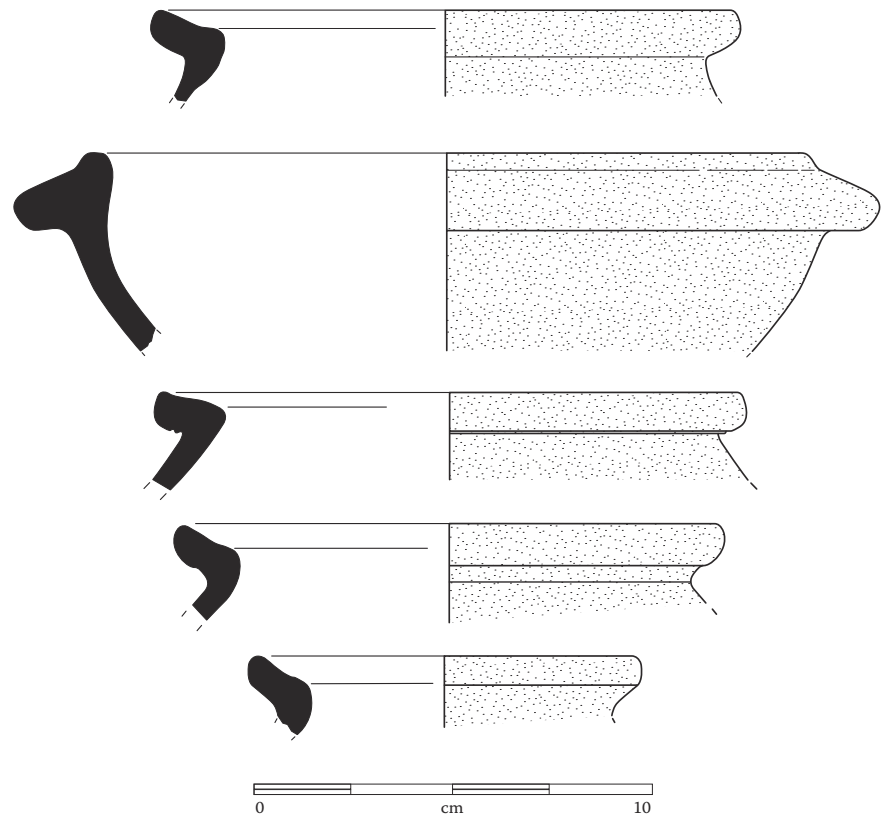
ponctuels. L'exploitation des carrières, si elle ne s'arrête pas, semble réduite au long de la période, alors même que se mettent en place des carrières pour la production de sarcophages. L'usage de remplois issus de grands appareils de construction antique pour la réalisation de sarcophages à partir du Bas Empire est fréquent et continu au moins jusqu'au tournant des $\mathrm{V}^{\mathrm{e}}-\mathrm{VI}^{\mathrm{e}}$ siècles (La Courneuve, Saint-Denis, Bondy).

La terre est fréquemment utilisée pour les hourdages dans la construction sur structure en bois. On retrouve régulièrement des traces de clayonnage dans les fragments de torchis recueillis en fouille. L'usage de la terre dans les couvertures est attesté par l'empreinte de chaume sur certains fragments. Les mélanges varient selon les usages : les hourdages sont constitués d'un mélange plus gras avec un proportion de paille élevée, les parois et soles de fours sont généralement plus chargées en sable et moins en végétaux. Le plâtre extrait de façon importante fournit principalement les ateliers de production de sarcophages. L'étude de ces contenants montre qu'ils relèvent d'une économie complexe : on observe la coexistence quasi systématique des sarcophages de pierre et de plâtre, ainsi que la présence récurrente de tombes maçonnées et de fosses simplement plâtrées. Le plâtre est aussi utilisé comme liant. Il est quelquefois, comme à La Courneuve, attesté en hourdage dès l'époque mérovingienne, mais aucune observation ne permet pour le moment d'extrapoler les usages constructifs du plâtre à l'habitat avant l'époque carolingienne. Les traces reconnues de plâtre dans la construction civile ne remontent qu'au $\mathrm{IX}^{\mathrm{e}}$, puis au $\mathrm{XI}^{\mathrm{e}}$ siècle. Les mortiers de chaux semblent raréfiés compte tenu des modes de constructions, toutefois leur usage est attesté à Villemomble et à Saint-Denis.

Les bâtiments en bois sont des structures sur poteaux et clayonnage combinés. Ce système constructif permet l'usage indifférencié ou associé du torchis et du plâtre pour les hourdages (La Courneuve VII ${ }^{\mathrm{e}}$-VIII ${ }^{\mathrm{e}}$ siècle, Saint-Denis VIII ${ }^{\mathrm{e}}$ $\mathrm{IX}^{\mathrm{e}}$ siècle, Tremblay-en-France $\mathrm{XI}^{\mathrm{e}}$ siècle...). L'absence notable de bâtiments sur poteaux sur les sites du département semble due aux phénomènes érosifs. Mais la récurrence de fragments de torchis et l'absence de maçonnerie laissent penser que ce type de construction était en usage. En ce qui concerne l'usage du bois de couverture, au moins un bardeau a été trouvé dans les combles de l'église de Noisyle-Grand en $2 \mathrm{O}^{2}{ }^{2}$. La couverture végétale, en chaume ou roseau reste certainement la plus fréquente.

Il est de coutume de considérer la persistance de l'usage de la tegula de tradition romaine jusqu'à l'époque carolingienne, même si on ne sait rien de cette production $\mathrm{du} \mathrm{VI} \mathrm{I}^{\mathrm{e}}$ au $\mathrm{IX}^{\mathrm{e}}$ siècle et aucun usage de brique n'est attesté sur le département à l'heure actuelle. On note cependant encore des phénomènes de remplois, en particulier de claveaux de voûte dans des fours (site de la rue Jules Guesdes à Tremblay-en-France, $\mathrm{VI}^{\mathrm{e}}$ - $\mathrm{VIII}^{\mathrm{e}}$ siècle).
Études spécifiques : céramique et faune

La céramique issue des sites du haut Moyen Âge en Seine-Saint-Denis ne présente pas de particularités au regard des sites du Nord de la France. À noter néanmoins, la découverte dans une fosse, sur le site de la route de Roissy, allée des Tilleuls à Tremblay-en-France, d'un tesson de céramique relevant des productions dites de Tating, céramique d'importation qui jalonne les grands itinéraires des marchands frisons et saxons caractérisée par sa pâte fine, sa surface noire et lustrée et son décor blanchâtre, obtenu par l'application de fines feuilles d'étain. Biens que rares, d'autres tessons de Tating ont été identifiés à Saint-Denis (Meyer-Rodrigues, 1993, p. 267-274). Cette découverte soulève des interrogations quant au statut de Tremblay-en-France au VIII ${ }^{\mathrm{e}}$ siècle. L'étude céramologique apporte surtout des informations importantes sur une période de transition peu documentée, la fin de l'époque carolingienne et le début de l'époque médiévale ( $\mathrm{X}^{\mathrm{e}}-\mathrm{XI}^{\mathrm{e}}$ siècles). En effet, nombre des habitats ruraux du haut Moyen Âge sont abandonnés durant cet intervalle. Les sites de Tremblay-enFrance permettent notamment d'appréhender ces évolutions [ill.3].

La plupart des sites mérovingiens de la SeineSaint-Denis s'inscrivent dans la catégorie des sites caractérisés par une proportion de bovins comprise entre 60 et $80 \%$ du cheptel domestique ${ }^{3}$. Les troupeaux sont alors majoritairement constitués d'individus mâles âgés. L'exploitation de la force motrice, destinée aux travaux de labours, est clairement privilégiée. L'animal est au service d'une agriculture extensive. Pour les phases carolingiennes, les sites ne se démarquent pas des autres contextes carolingiens du pays de France avec une proportion de bœufs, dans le cheptel domestique, supérieure à $50 \%$. Seul le site de la rue Louis-Delplacé à Drancy (Gonçalves 2003a ; Gonçalves-Buissart et al., 2011) pourrait se $32 \%$ et d'une part importante de caprinés, mais la faiblesse du nombre de restes n'autorise pas une interprétation fiable.

\section{Modes d'organisation de l'habitat et formation des paroisses}

Durant l'Antiquité, le département a connu un peuplement caractérisé par un habitat dispersé composé de grandes exploitations, les villae, mais également d'habitats secondaires plus nombreux. Ceux-ci sont attestés principalement sur les plateaux. Bien que souvent implantés à courte distance de ces sites, les habitats se fixent, à partir $d u v^{\mathrm{e}}$ siècle, dans les vallées. Le faible nombre de ces villae et leur état de conservation ne permettent pas d'évaluer leur rôle éventuel dans la transition entre la fin de l'Antiquité et le début du Moyen Âge. Les villages du haut Moyen Âge, en Seine-SaintDenis, ne se sont, a priori, pas développés aux abords d'établissements antiques ; il ne semble donc pas distinguer du fait d'une proportion de seulement

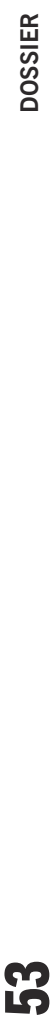

ஜ 


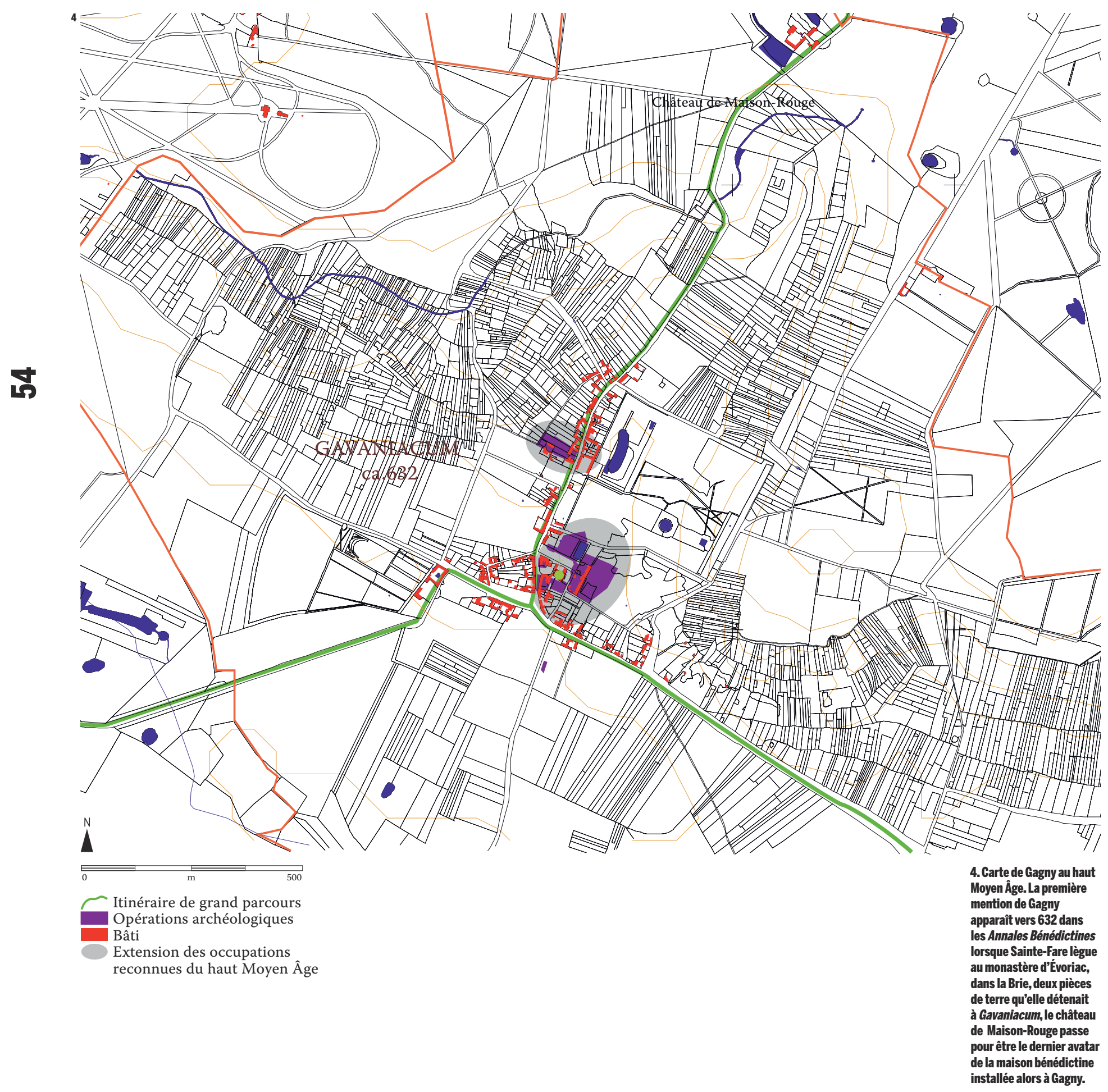


y avoir de filiation directe entre les deux formes d'habitat. Les fouilles attestent presque exclusivement de fondations de villages à la période mérovingienne voire carolingienne. Ầ l'échelle de la Seine-SaintDenis, si une organisation globale des habitats est encore difficile à dégager des observations archéologiques, on peut émettre quelques hypothèses.

\section{Habitats structurés par les axes routiers}

Les différentes occupations alto-médiévales attestées se concentrent notamment en bordure de quatre axes importants de circulation. À Bondy", là où l'itinéraire Seine-Marne rejoint celui de Paris à Meaux (l'actuelle RN3), une occupation significative de la fin de l'Antiquité et du début du haut Moyen Âge, notamment connue par la fouille en 20052006 d'une importante nécropole et par la mention, dans le testament d'Erminthrude, au VI ${ }^{\mathrm{e}}$ ou VII ${ }^{\mathrm{e}}$ siècle, de l' «ecclisiae vici Bonisiacinsis » (Atsma, Vezin, 1985). L'actuelle RN 186 est jalonnée par plusieurs sanctuaires attestés à l'époque mérovingienne: Saint-Rémy à Saint-Denis (dont l'habitat et le cimetière ont été partiellement fouillé par l'Inrap et l'UASD entre 1996 et 2009), SaintLucien à La Courneuve [ill.5], Saint-André à Bobigny dont le domaine est mentionné dans le testament d'Erminthrude (Atsma, Vezin, 1985) et Saint-Pierre à Bondy. À Gagny, un important habitat s'est installé à la jonction de deux axes anciens ${ }^{\mathbf{5}}$ : la rue des Carneaux et la rue allant à Montfermeil [ill.4]. Enfin de nombreux sites ont été identifiés de part et d'autre de la route allant de Roissy à Tremblay-en-France et de la route allant de Tremblay à Villepinte.

\section{Habitats organisés autour des cours d'eau}

Le regroupement d'habitat autour d'un cours d'eau semble prévaloir avant la fixation définitive du réseau paroissial, entre le haut Moyen Âge et le $\mathrm{XI}^{\mathrm{e}}$ siècle. On l'observe très nettement à Tremblay et de manière un peu plus diffuse mais avec récurrence à Saint-Léger de Gassenville, entre Stains et SaintDenis (Wyss,1996), à Gagny, à Savigny près d'Aulnay .

Aux abords de Saint-Denis, dans la gouttière alluviale du Croult, à Stains et La Courneuve, on observe la mise en place d'un réseau d'habitat rural mérovingien associé aux cours d'eau (Rouillon, Vieille Mer, dérivation du Croult et ru de Montfort). L'habitat du haut Moyen Âge semble s'organiser aux abords directs du Rouillon, réparti de distance en distance (3 sites se répartissent sur environ $1,5 \mathrm{~km}$, correspondant approximativement à une demi-lieue (Lafarge, 2003b ; Leconte, 2004; Wyss, 1996). À Saint-Léger ${ }^{7}$ quelques tombes se révèlent clairement appartenir à un cimetière paroissial primitif, bien attesté et associé à un habitat tout proche, mais aussi dispersé dans le fond de vallée en remontant le cours du ruisseau ${ }^{\mathbf{8}}$. Il est possible que l'habitat de Merville, aujourd'hui sous le parc paysager de la Courneuve appartienne à ce groupement (Wyss, 1996). Durant le haut Moyen Âge, ces habitats semblent se rattacher à la paroisse de Saint-Léger de Gassenville mentionnée comme Vasconsis villa en 832 dans le partage des biens de Saint-Denis par l'abbé Hilduin. Cette paroisse est fondée vers la fin du VII ${ }^{\mathrm{e}}$ siècle. Les différentes occupations semblent péricliter entre les $\mathrm{X}^{\mathrm{e}}$-XI ${ }^{\mathrm{e}}$ siècles et 1213. Les occupations attestées du haut Moyen Âge apparaissent comme étendues et lâches à la fois; il est possible que ces établissements aient eu des occupations relativement courtes et se soient déplacés d'autant plus fréquemment.

\section{Occupations de plateau}

À Tremblay-en-France, les sites dispersés autour du Sausset tendent à se regrouper autour d'un pôle marqué par la seigneurie de l'abbaye de Saint-Denis (Lafarge, Rémy, 2011). En 862, la confirmation par Charles le Chauve du partage des biens de l'abbaye de Saint-Denis entre mense abbatiale et mense conventuelle mentionne les villae de Tremblay et de Villepinte. Cela implique l'existence d'une population dont les fouilles montrent les traces d'habitation.

Les sites du Château Bleu, de la rue Jules Guesde et de « l'imprimerie du Figaro » autour du Petit Tremblay, au nord, sont des points de concentration de l'habitat à partir de l'époque mérovingienne. Il s'agit de groupements de petites exploitations rurales qui se développent jusqu'au $\mathrm{x}^{\mathrm{e}}$ siècle. $\mathrm{Au} \mathrm{XI}{ }^{\mathrm{e}}$ siècle, le site du Château Bleu s'apparente plutôt aux enceintes paysannes.

Au Grand Tremblay, la fouille rue des Tilleuls, évoque les mutations de l'espace qui deviendra le village depuis le $\mathrm{V}^{\mathrm{e}}$ siècle [ill.6]. L'occupation commencée dès la fin du $\mathrm{v}^{\mathrm{e}}$ siècle se développe autour du carrefour de la route de Roissy et de la rue Cruppet, aboutissement du chemin de Villepinte à Tremblay. Le site se structure en petites unités séparées par des petits fossés garnis de haies ou de palissades formant des parcelles longues et étroites orientées est-ouest. La plupart des structures se rapportent à des activités agraires, artisanales ou de stockage. Ces sites forment un groupement lâche au centre duquel sont installés sur une légère butte, mais à une date encore indéterminée, l'église Saint-Médard, puis l'habitat seigneurial. Si l'installation de l'église n'est pas encore précisément datée, on sait que le cimetière qui lui est associé remonte à l'époque mérovingienne. Pour l'habitat seigneurial, la seule certitude est son existence $\mathrm{au} \mathrm{IX}^{\mathrm{e}}$ siècle. Une permanence dans le parcellaire existe depuis le haut Moyen Âge, mais de nombreuses divisions semblent avoir disparu au tournant des $\mathrm{XII}^{\mathrm{e}}$ et XIII ${ }^{\mathrm{e}}$ siècles. À partir du XIII ${ }^{\mathrm{e}}$ siècle, des bâtiments sur poteaux sont installés en bordure de la Grande Rue (route de Roissy) qui longe le site à l'ouest. Des constructions maçonnées ne sont installées qu'à partir du XIII ${ }^{\mathrm{e}}$ siècle.

Ces ensembles de groupements d'habitations révèlent bien une organisation générale en unités comprenant des éléments « habituels » des 


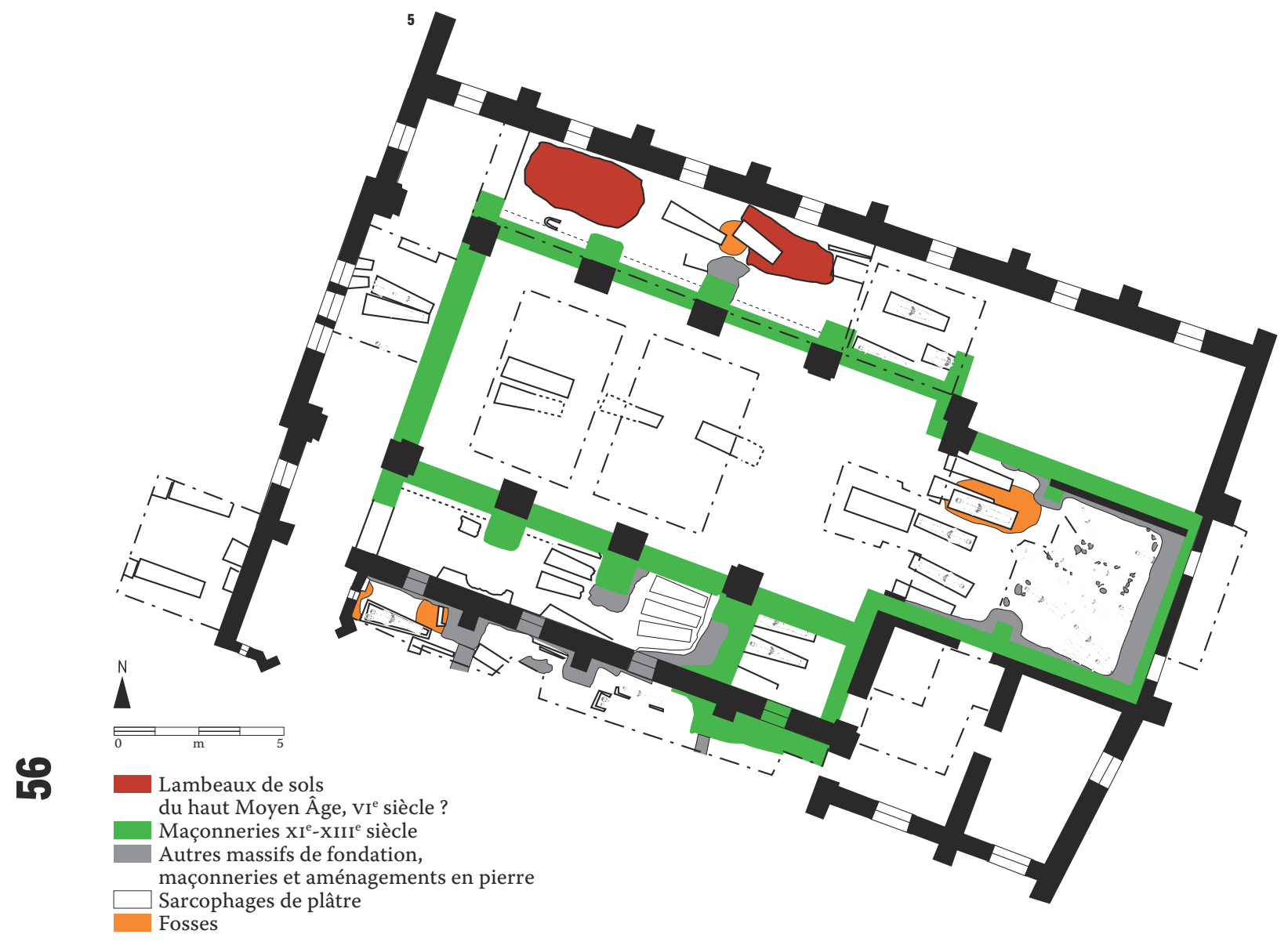

5. Plan compilé des

données archéologiques

sur l'église Saint-Lucien

à La Courneuve recueillies

de 1973 à 1981 par

A. Bulard, J.J. Charpy,

M.C.Godet et C. Gaborieau.

6. Vue aérienne du Vieux Pays de Tremblay-en-

France.

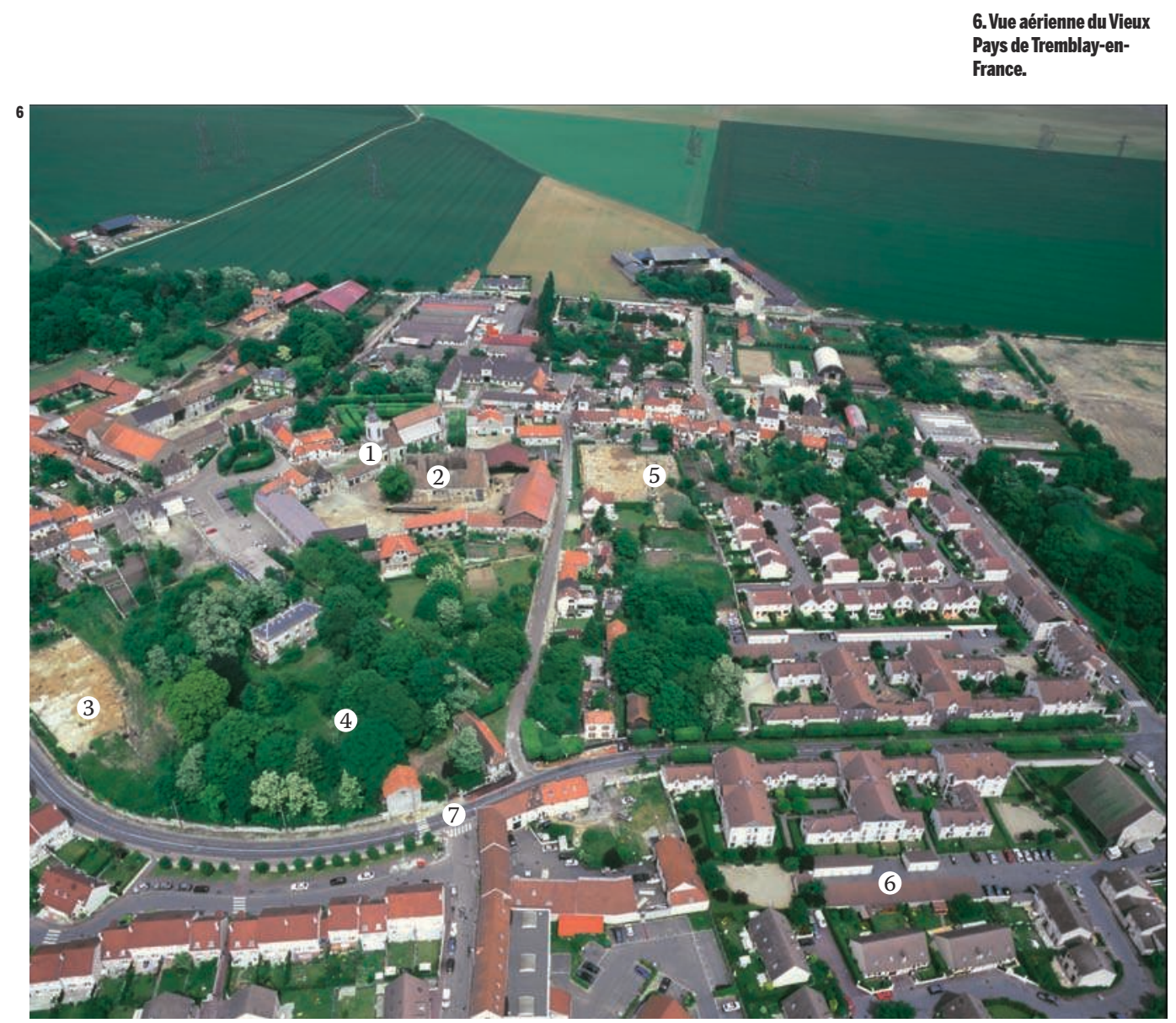

1. Église Saint-Médard.

2. Grange aux dîmes.

3. et 4. Fouilles de l'allée des Tilleuls

(1998 et 2007).

5. Fouille de la rue des Fossés.

6. Fouille de la rue Cruppet.

7. Route de Roissy. 
occupations rurales du haut Moyen Âge : maisons et annexes comprenant greniers, silos, batteries de fours, fonds de cabanes associés à des systèmes d'enclos (ou de haies) souvent discontinus où il n'est pas toujours aisé de démêler les éléments communautaires des éléments relevant de la sphère privée. Évidemment ces schémas ne sont pas absolus certains sites semblent se rattacher à d'autres modes d'occupation du terroir, ainsi, à Montreuil, à Villemomble, ou à Noisy-le-Grand. À Drancy, un schéma similaire paraît se développer à cette différence que l'habitat dispersé n'encadre pas de cours d'eau. Installés dans un paysage largement ouvert, les habitats mérovingien et carolingien montrent des traces d'aménagements agricoles et artisanaux, associés à un habitat dispersé au sein d'enclos juxtaposés.

Sur certains terroirs, l'habitat reste parfois encore un fait problématique, ainsi à Noisy-le-Grand où la mention par Grégoire de Tours d'une villa regia, l'existence d'une importante nécropole au lieu-dit «Les Mastraits », connue depuis 1771 (dont 651 tombes du VI ${ }^{\mathrm{e}}$ au XII ${ }^{\mathrm{e}}$ siècle ont été fouillées en 2009-2010 dans une emprise qui ne semble représenter que $1 / 3$ à $1 / 2$ de la surface de la nécropole), tout porte à croire à l'existence d'un important habitat, pas encore localisé. À Villemomble, une importante nécropole a été mise au jour dans les années 1980 (Delahaye). Même si la zone d'inhumation n'a pas été intégralement reconnue, c'est un des rares endroits où une église d'époque mérovingienne ait pu être mise au jour, mais l'habitat n'est pas connu à l'heure actuelle.

À partir des $\mathrm{XI}^{\mathrm{e}}-\mathrm{XII}^{\mathrm{e}}$ siècles, on observe la cristallisation de villages au détriment d'un habitat rural essentiellement constitué de groupements épars. Dans cette mutation, le réseau paroissial et la répartition de la seigneurie foncière jouent un rôle déterminant mais complexe, en sorte que les agglomérations villageoises ne sont pas nécessairement accolées aux églises ou aux sites seigneuriaux. De la constitution des villages, il découlera progressivement la généralisation de constructions maçonnées au détriment de la construction en bois et torchis.

\section{Références bibliographiques}

Astma H., VeZin J., 1985, «Le testament d'Erminthrude», in PERIN P. (DIR.), Collections mérovingiennes; Catalogues d'art et d'histoire du musée Carnavalet II, Paris, imprimerie municipale, $864 \mathrm{p}$.

GonçALVES C., 2003a, « Notice de site : Drancy (SeineSaint-Denis) "rue Louis Delplacé, rue de la Poterie" », in LefÈvre A., Gentili F., MAhÉ N. (DIR.), p. 56-63.

GonÇALVES C., 2003b, «Notice de site: Tremblay-enFrance (Seine-Saint-Denis) "rue Louis Eschard, allée de la Mairie" », in Lefèvre A., Gentili F., Mahé N. (DIR.), p. 72-74.

GONCALVES C., 2003C, "Notice de site : Tremblay-enFrance (Seine-Saint-Denis) "Allée des Tilleuls, route de Roissy" », in Lefèvre A., Gentili F., Mahé N. (DIR.), p. 75-78.

GonCAlves C., 2003d, « Notice de site: Tremblay-enFrance (Seine-Saint-Denis) "Château Bleu" », in Lefèvre A., Gentili F., MahÉ N. (Dir.), p. 78-81.

GonÇALVES-Buissart C., 2009, « Notice de site: Tremblay-en-France (Seine-Saint-Denis) "Les Ruisseaux, Route départementale 40" ", in LefÈvre A., GeNTILI F., MAHÉ N. (DIR.), p. 257-270.

Gonçlves-Buissart C., Héron C., Le Béchennec Y., Haye F. (COllab.), C. Munoz (Collab.), 2011,

« Drancy (Seine-Saint-Denis), de La Tène à l'époque Moderne : état des connaissances ", Revue archéologique d'Ile-de-France, n ${ }^{\circ}$ 3-2010, p. 177-219.

Goret J.-F., 2009, Saint-Denis (Seine-Saint-Denis) 3, rue du Général-Joinville, rapport de fouille préventive, UASD, $75 \mathrm{p}$.

LAFARGE I., 2003a, « Notice de site : Gagny (Seine-SaintDenis) "Rue Aristide Briand et du Clos Félix" », in Lefèvre A., Gentili F., Mahé N. (Dir.), p. 64-66.

LAFARGE I, 2003b, «Notice de site: Stains (Seine-SaintDenis) "Site Hoechst-Duco, Le Moulin Neuf" », in Lefèvre A., Gentili F., Mahé N. (DIR.), p. 67-68. 